\title{
Assessment of efficacy and safety of the herbal medicinal product BNO 1016 in chronic rhinosinusitis*
}

\author{
J. Palm', I. Steiner², D. Abramov-Sommariva33, A. Ammendola³, \\ S. Mitzenheim ${ }^{3}$, H. Steindl ${ }^{3}$, M. Wonnemann ${ }^{3}$, C. Bachert ${ }^{4}$, \\ on behalf of the study group \\ ' Röthenbach, Germany \\ 2 Posnan, Poland \\ 3 Bionorica SE, Neumarkt, Germany \\ ${ }^{4}$ Ghent University Hospital, Ghent, Belgium
}

Rhinology 55: 142-151, 2017

https://doi.org/10.4193/Rhino16.103

*Received for publication:

March 14, 2016

Accepted: January 11, 2017

\begin{abstract}
Background: The objective of this clinical trial (CRS-02) was to assess the efficacy, safety and tolerability of two dosages of the herbal medicinal product BNO 1016 (Sinupret extract) in patients with chronic rhinosinusitis (CRS).

Methodology: 929 patients suffering from CRS were enrolled in this randomised placebo-controlled trial with a treatment period of 12 weeks. The primary endpoint was the mean Major Symptom Score (MSS) in week 8 and week 12 compared to placebo. Secondary endpoints included further MSS related parameters and responder rates over time. Pharmacoeconomic endpoints were also analysed. Finally, safety and tolerability were evaluated.
\end{abstract}

Results: Sinupret extract was not superior over placebo regarding the primary endpoint. However, the results of the secondary endpoints showed a clear trend towards superior efficacy. Therefore, additional post-hoc sensitivity analyses were performed in patients with a baseline MSS > 9 and persistence of disease $>1$ year diagnosed by specialists in otorhinolaryngology. Those patients significantly benefited from Sinupret extract. Therapy was superior for the primary endpoint analysis. Patients were less impaired with respect to work and daily activities. A good safety and tolerability of Sinupret extract was assured in all patients.

Conclusions: Sinupret extract can safely be administered in patients with CRS. Although the primary endpoint of the study was not significant, a post-hoc subgroup analysis in patients whose disease was diagnosed by a specialist revealed a pronounced treatment effect. Effects in that subgroup were even stronger with longer disease persistence and stronger severity.

Key words: BNO 1016, Sinupret extract, herbal medicine, rhinosinusitis, chronic, EPOS 2012

\section{Introduction}

Rhinosinusitis is an inflammation of the nose and the paranasal sinuses. Diagnosis is mainly based on signs and symptoms; symptoms like nasal blockage or nasal discharge together with facial pain, facial pressure, reduction or loss of smell characterize the disease. It is considered chronic when symptoms persist for more than twelve weeks. It may additionally reveal signs of polyps, mucopurulent discharge, oedema or mucosal obstruction when performing a nasal endoscopy. Furthermore, computer tomography might show mucosal changes within the ostiomeatal complex or the sinuses ${ }^{(1)}$.
Etiology of the disease remains a matter of debate. It is believed that chronic rhinosinusitis (CRS) may develop from acute infections. The mechanisms and cofounders of transition from acute to chronic rhinosinusitis are still under research, but are discussed to involve smoking, bacterial or fungal infections, innate and adaptive immune dysfunction or allergies ${ }^{(1,2)}$. Recent findings also support a relation to patients with severe chronic upper airway diseases and suggest neurogenic and systemic pathways ${ }^{(3)}$.

According to EPOS 2012 guideline (European position paper on rhinosinusitis and nasal polyps $2012^{(4)}$ ) and Hastan et al. ${ }^{(5)}$, 
the overall prevalence of CRS may be estimated as around $11 \%$ (range $7-27 \%$ ) of the general population in Europe and considerably impacts physical constitution and quality of life. It produces direct costs through consultation visits and antibiotics prescriptions as well as indirect costs related to days off work and a general loss of productivity ${ }^{(6,7)}$. CRS therefore constitutes a major burden for the health care system. Some relief from congestive symptoms can be achieved by application of nasal saline spray, nasal lavage with isotonic saline or steam inhalation. Besides, pharmacological treatment of CRS includes antibiotics, local decongestants, nasal steroids and herbal medicines like myrtol or cineol.

BNO 1016 had been established for use in patients with acute rhinosinusitis and is marketed under the brand name 'Sinupret extract CT'. It contains the dry extract of five herbal drugs, namely gentian root, primula flower, sorrel herb, elder flower, verbena herb, in a ratio of 1:3:3:3:3. The efficacy in acute rhinosinusitis has been demonstrated in a randomised, placebo-controlled trial ${ }^{(8)}$. In this clinical trial Sinupret extract was applied three times daily at a dose of $160 \mathrm{mg}$ each. In this study (ARhiSi-2), Sinupret extract demonstrated to be clinically and significantly superior versus placebo in terms of reduction of symptoms as measured by the reduction in the Major Symptom Score (MSS; $p=0.008)$. Furthermore, the proportion of patients having been cured (MSS $\leq 1$ ) was significantly higher in the active treatment group than in the placebo group (48.4\% vs. $35.8 \%$; $p=0.0063$ ). Additionally, more patients were free of signs of rhinosinusal inflammation in the ultrasound investigation $(73.2 \%$ vs. $61.6 \%$; $\mathrm{p}=0.0131)^{(8)}$.

The tolerability of Sinupret extract was comparable to that of placebo. About $95 \%$ of the patients rated tolerability to be 'very good' or 'good' (8).

The present study CRS-02 extended the efficacy evaluation to the indication of chronic rhinosinusitis.

\section{Materials and methods}

\section{Study design}

The CRS-02 study was designed as a multicentre, randomised, double-blind, placebo-controlled, parallel group trial to assess efficacy as well as safety and tolerability of two dosages of Sinupret extract in patients with CRS. It was conducted from September 2012 to April 2014 in 67 trial centres in Belgium, Czech Republic, Germany and Poland. The investigations were led by specialists in otorhinolaryngology as well as internal medicine and general practitioners. Trial participants passed through the study with ambulatory treatments. A hospitalisation did not take place. The study included a screening period of up to 2 weeks (visit 1), a 12-week treatment period (visit 2 to 6) and an 8-week follow-up period (visit 7). Patients were randomised at day 1 (visit 2) and control visits were scheduled after 1 week (visit 3), 4 weeks (visit 4), 8 weeks (visit 5), and 12 weeks (visit 6). Follow-up examination was scheduled 8 weeks after visit 6 , adding up to a total trial duration of 20 weeks.

The study was conducted in accordance with the Declaration of Helsinki ${ }^{(9)}$ and the ICH Guideline for Good Clinical Practice ${ }^{(11)}$. It was also approved by the relevant competent authorities of the participating countries and received a favorable opinion by the competent ethics committees. Written informed consent was obtained from all patients prior to inclusion.

\section{Patients}

In the CRS-02 study, adult male and female outpatients with the following criteria were eligible: 18 to 75 years of age with bilateral CRS without nasal polyps confirmed by endoscopy (optional computer tomography not older than 2 years). Symptoms had to be present for at least 12 weeks prior to enrolment without complete resolution. Total MSS score ${ }^{(8,10)}$ had to be 6 to 12 points and rhinorrhea and pain (facial pain or headache) had to be of at least moderate intensity (MSS score at least 2).

Exclusion criteria were: sinus surgery during the past 2 years, CRS due to allergic origin, treatment with systemic or nasal antibiotics or corticosteroids within the last 4 weeks prior to inclusion and patients treated with decongestant preparations, analgesics, mucolytics/secretolytics, antihistamines, or alternative medicine preparations for treatment of common cold like symptoms or with immunomodulating drugs within the last 7 days prior to inclusion.

\section{Treatments}

At baseline (visit 2), patients were randomised in a 1:1:1 ratio to receive either Sinupret extract $240 \mathrm{mg}$ per day, $480 \mathrm{mg}$ per day or placebo in a double-blind mode. Sinupret extract was provided as coated tablets containing $80 \mathrm{mg}$ of the dry extract of the five herbal drugs. Study medication was administered orally. Each patient received two tablets three times daily, either Sinupret extract $80 \mathrm{mg}$, matching placebo or a combination of both.

\section{Assessments}

At each visit, patients were evaluated by the investigator for the five symptoms of the Major Symptom Score (MSSINV). These are rhinorrhea/anterior discharge, postnasal drip, nasal congestion, headache, and facial pain or pressure. Patients recorded symptoms in a diary (MSS ${ }^{\mathrm{PAT}}$ ) at five days during the screening period, while days could be chosen in an arbitrary way. Moreover, they recorded symptoms on a daily basis between visit 5 (week 8) and 6 (week 12). The severity of each of the five symptoms was indicated on a 4-point rating scale of increasing severity $(0=$ none; $1=$ mild; $2=$ moderate; $3=$ severe $)$. The MSS was calculated as the sum of the five individual symptom scores. Patients and investigators assessed the response to treatment 
at visit 3 (week 1) to 6 (week 12) based on a 4-point rating scale $(0=$ symptom healed; 1 = symptom improved; 2 = symptom unchanged; 3 = symptom deteriorated). Patients with healed or improved symptoms were classified as responders.

The participants assessed the total symptom severity on a visual analogue scale at visit 2 (day 1) to visit 7 (week 20) with $0 \mathrm{~mm}$ meaning 'not troublesome' and $100 \mathrm{~mm}$ meaning 'worst thinkable troublesome'. The Sino-Nasal Outcome Test-20 (SNOT20) in the German adapted version (SNOT-20 GAV) was used to evaluate the impact of rhinosinusitis symptoms on healthrelated quality of life (HRQoL). Patients rated the severity of the 20 questionnaire items retrospectively for the last 24 hours at baseline and at each post-baseline visit using a 0-5 point Likert scale $(0=$ not present $/$ no problem; $1=$ very mild problem; 2 $=$ mild or slight problem; $3=$ moderate problem; $4=$ severe problem; 5 = problem as bad as it can be). The SNOT-20 GAV was used only in German centres.

At each visit starting from day 1 , patients were asked to report any unscheduled physician consultation for CRS-related symptoms, CRS-related days off work or missed daily activities since the last visit. Participants answered the Work Productivity and Activity Impairment: General Health (WPAl:GH) questionnaire to measure impairments in work and activities during the previous week.

Safety and tolerability were evaluated based on the frequency and severity of adverse events, measurement of vital signs and analysis of laboratory parameters and physical examinations. Patients and investigators were asked to judge the tolerability at the end of the treatment period using a 5-point rating scale ranging from 0 ('very good') to 4 ('very poor').

\section{Endpoints}

The primary endpoint was the mean MSS assessed by the investigator (MSS ${ }^{\mathrm{INV}}$ ) at visit 5 (week 8) and visit 6 (week 12). A difference of $\Delta 0.80$ score points in the primary endpoint between the active treatment (high and low dose) and placebo was considered to be clinically relevant. In the preceding clinical trial in acute rhinosinusitis ${ }^{(8)}$ a minimal clinical important difference of $\Delta 1.0$ score points was chosen and confirmed by otorhinological experts. However, in contrast to an acute state of the disease, improvements in the chronic state are generally slower and not as conspicuous for the patient and the investigator. Therefore, $\Delta$ was reduced to 0.8 which was again confirmed by the experts. The investigator based score was chosen following a former decision of the competent German authority on the corresponding clinical trial in acute rhinosinusitis. Patient based evaluations were included as secondary efficacy endpoints ${ }^{(8)}$. Major secondary efficacy endpoints included the mean MSS ${ }^{\text {PAT }}$ at visit 5 (week 8) and visit 6 (week 12), change of mean MSSINV over time on each visit starting from visit 3 (week 1) until visit 7 (week 20), discrete and mean symptom scores for single MSS items assessed by the investigator and the patient, the mean severity of single symptoms and responders rates over time.

Pharmacoeconomic endpoints were defined as the number of days off work or missed daily activities, the percentage of work time missed, the percentage of impairment while working due to health, the percentage of overall work impairment and the percentage of activity impairment due to health (all taken from the WPAl:GH-questionnaire. Finally, safety was analysed as the number of patients with adverse events, the number of patients by tolerability score and regarding changes in vital signs and laboratory parameters.

\section{Statistical methods}

A pre-planned interim analysis was performed in March 2013 to re-estimate sample size. The interim analysis used the efficacy data of 375 patients without unblinding treatment assignment. Based on the updated variance data for the primary endpoint, the sample size had been increased from 771 to 900 patients. The analyses of the primary and secondary endpoints were using the full analysis set (FAS) which included all randomised patients with at least one administration of study drug and available post-baseline efficacy data. The safety population described by the safety evaluable set (SES) included all randomised patients with at least one documented administration of trial medication and any post-baseline safety data. The per protocol set (PPS) was additionally evaluated as sensitivity analysis.

Moreover, post-hoc sensitivity analyses considered patients with a baseline MSS > 9, patients recruited by otorhinolaryngologists and patients with a persistence of CRS $>1$ year.

The primary efficacy endpoint was tested using a superiority hypothesis versus placebo employing ANCOVA with 'MSS at Baseline' as a fixed effect covariate and 'Treatment' as a fixed effect. The centre effect described by the variable 'Medical Specialist" was included in the model as fixed effect. The Null hypotheses were tested with a one-sided type-I error rate of $2.5 \%$ (adjusted for two confirmatory tests according to Benjamini-Hochberg) ${ }^{(12)}$. Secondary endpoints were analysed in an explorative manner. Standard descriptive statistics were used for the analysis of secondary efficacy endpoints, pharmacoeconomic endpoints and safety data. Continuous variables were described by median and mean values. Standard deviation, quartiles, minimum and maximum were used as indices of variability. Categorical variables were described as absolute numbers and percentages. All data were analysed using the software package SAS release 9.2 (SAS Institute Inc., Cary, NC, USA) ${ }^{(13)}$. Missing efficacy values except for the SNOT-20 GAV analysis were handled by a mixed model for repeated measures that assumes that missing values are'missing at random' (MAR) (primary estimate). A sensitivity analysis was included, to assess the robustness of the primary estimate with regard to missing data. MAR assumptions were replaced by assumptions that were likely to be relatively less favorable 


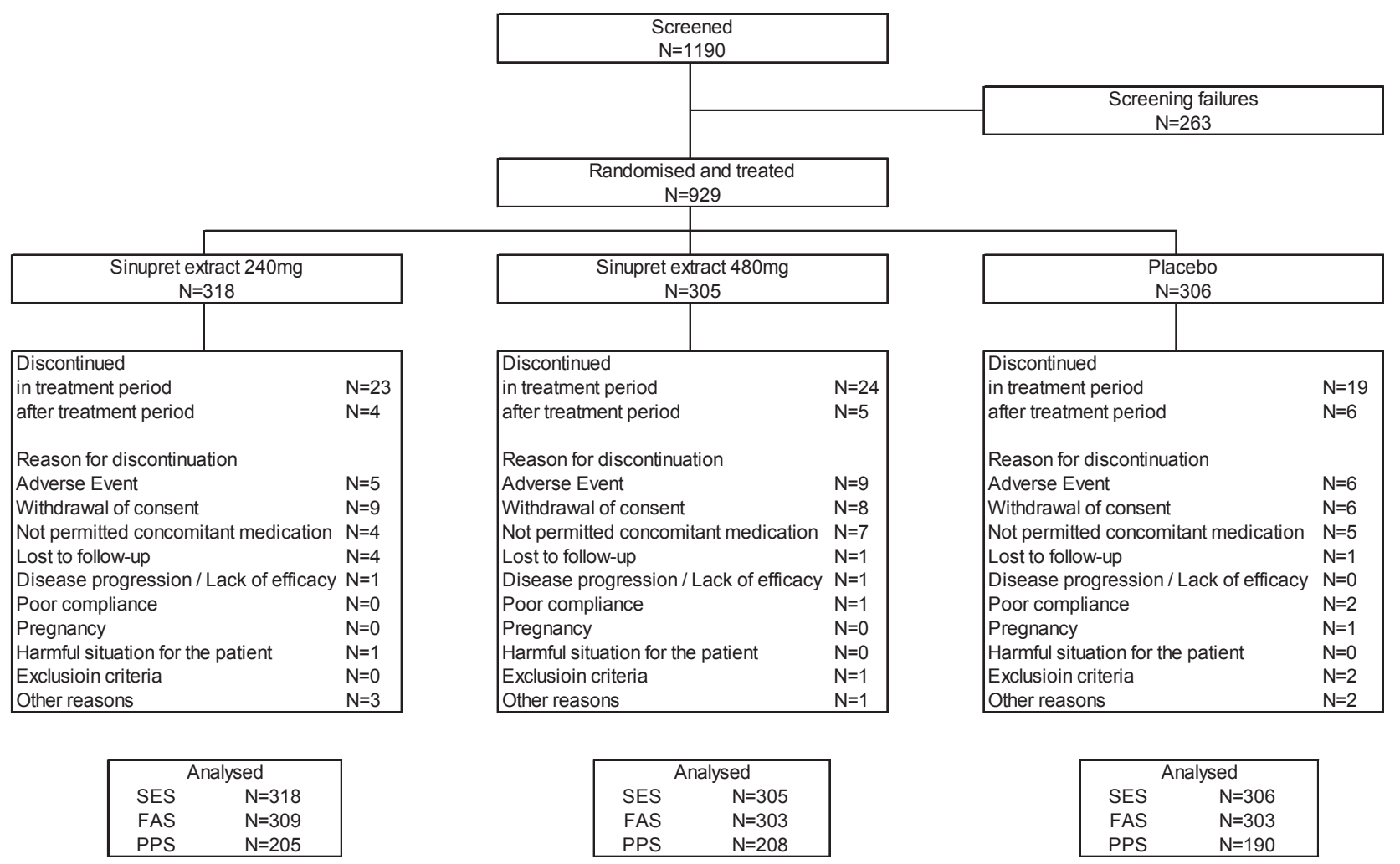

Figure 1. Patient disposition.

to the treatment or in case of SNOT-20 GAV by worst category as long as only one item per subscore was missing. All reported probability $(p)$ values in this publication are two-sided $p$-values unless otherwise specified.

\section{Results}

Patient disposition

The disposition of patients is displayed in Figure 1. In total, 929 patients were randomised and received investigational treatment ( 240 mg N=318; 480 mg N=305; placebo $N=306$ ). These patients constituted the SES. Fourteen patients were excluded from the efficacy analysis for major protocol violations (no post-baseline efficacy data available for 11 patients; emergency envelopes opened for 3 patients). The PPS consisted of 603 patients, i.e. 326 patients were excluded for 482 major protocol deviations.

The most common ( $>5 \%$ of all protocol violations) were:

- Unpermitted administration of paracetamol one day before visits 2,5 or 6 , i.e. before baseline, week 8 or week 12 (17.9\%)

- Failure to meet the inclusion criteria with respect to the intensity of rhinorrhoea and pain (11.7\%) or MSS severity (7.3\%)

- $\quad$ Antibiotic use during the 4 weeks prior to inclusion (7.9\%)

- Use of prohibited medication before or during the study

\section{(6.0\%)}

- $\quad$ Analgesic use within three days prior to visits 2, 5 or 6 , i.e. before baseline, week 8 or week 12 (5.6\%)

- Missing primary endpoint data at visit 5 (week 8) (5.4\%). Overall, 81 patients discontinued the study prematurely, 66 during the treatment period and 15 afterwards. The main reasons were non-tolerable adverse events $(\mathrm{N}=20)$, withdrawal of consent $(\mathrm{N}=23)$ and application of non-permitted concomitant medication ( $\mathrm{N}=16)$.

Assessment of the patients' demographic and baseline characteristics indicated that all groups were adequately balanced. Mean age was $44.3 \pm 14.8$ years and $61.4 \%$ of all patients were women. The median treatment duration was 85 days ( $\approx 12$ weeks).

\section{Efficacy results}

Baseline MSS ${ }^{\mathrm{INV}}$ scores on visit 2 (day 1) of the trial were 10.7 (240 $\mathrm{mg}), 10.7$ (480 mg) and 10.8 (placebo) score points ( $p>0.05, F A S)$. Treatment with Sinupret extract was not superior over placebo at the primary endpoint analysis with mean MSS ${ }^{\text {INV }}$ (averaged for visit 5 and 6, i.e. week 8 and week 12) of 5.1 ( $240 \mathrm{mg}$ ), 5.1 (480 $\mathrm{mg}$ ) and 5.3 (placebo) score points ( $p>0.05$, FAS). However, the results of the secondary efficacy endpoints indicated that daily treatment with Sinupret extract for 12 weeks was effective in improving symptoms and health related quality of life in patients with CRS. In general, all patient assessments were similar and reflected the results of the assessments done by the investigators. 
Table 1. Results of single items of the MSSINV in subgroup of patients who had a baseline MSS $>9$, had CRS persistence $>1$ year and were treated by a specialist in otorhinolaryngology (FAS).

\begin{tabular}{|c|c|c|c|c|}
\hline & $\begin{array}{c}\text { Sinupret extract } \\
240 \mathrm{mg}\end{array}$ & $\begin{array}{l}\text { Sinupret extract } \\
480 \mathrm{mg}\end{array}$ & Placebo & $\begin{array}{l}\text { p-value vs. Placebo } \\
240 \text { mg / } 480 \text { mg }^{\mathrm{a}}\end{array}$ \\
\hline \multicolumn{5}{|c|}{ Averaged MSS ${ }^{\prime N V}$ visit 2 (day 1) (baseline); Mean (SD) } \\
\hline & $\mathrm{N}=86$ & $\mathrm{~N}=93$ & $\mathrm{~N}=75$ & \\
\hline & $10.7(0.8)$ & $10.7(0.8)$ & $10.8(0.8)$ & \\
\hline \multicolumn{5}{|c|}{ Averaged MSS ${ }^{I N V}$ visit 5 (week 8) and visit 6 (week 12); Mean (SD) } \\
\hline & $\mathrm{N}=86$ & $\mathrm{~N}=93$ & $\mathrm{~N}=75$ & \\
\hline & $6.3(2.7)$ & $5.3(2.8)$ & $6.8(3.4)$ & $0.3224 / 0.0015$ \\
\hline \multicolumn{5}{|c|}{ Averaged single MSS ${ }^{I N V}$ items visit 5 (week 8) and visit 6 (week 12); Mean (SD) } \\
\hline & $\mathrm{N}=86$ & $\mathrm{~N}=93$ & $\mathrm{~N}=75$ & \\
\hline Rhinorrhea anterior & $1.3(0.6)$ & $1.0(0.7)$ & $1.5(0.8)$ & $0.1080 /<0.001$ \\
\hline Rhinorrhea posterior & $1.6(0.6)$ & $1.3(0.7)$ & $1.5(0.8)$ & $0.6691 / 0.1392$ \\
\hline Nasal congestion & $1.4(0.7)$ & $1.2(0.8)$ & $1.4(0.8)$ & $0.9566 / 0.1827$ \\
\hline Headache & $1.0(0.7)$ & $0.8(0.7)$ & $1.2(0.9)$ & $0.2070 / 0.0101$ \\
\hline Facial pain & $1.1(0.7)$ & $0.9(0.7)$ & $1.2(0.8)$ & $0.1781 / 0.0009$ \\
\hline
\end{tabular}

a ANCOVA with 'MSS at Baseline (visit 2, day 1)' as a fixed effect covariate and 'Treatment' as a fixed effect. The centre effect described by the variable 'Medical Specialist' was included in the model as fixed effect.

Significant differences in favor of Sinupret extract were demonstrated for the investigator assessments from visit 3 (week 1) to visit 7 (week 20) for rhinorrhea, headache and facial pain/pressure. Patients assessed facial pain/pressure and the total symptom severity as significantly improved compared to placebo.

As assessed by the WPAI:GH, patients treated with Sinupret extract had a significantly greater improvement compared to placebo-treated patients regarding the outcome 'percent impairment while working due to health' at visit 7 (week 20), and regarding the outcome 'percent overall work impairment due to health' at visit 5 (week 8) and visit 7 (week 20). Finally, no treatment effect was seen regarding the other outcomes generated from the WPAI:GH ('percent work time missed due to health' and 'percent activity impairment due to health').

\section{Post-hoc sensitivity analysis}

Subgroup analyses on the clinical data obtained were first performed considering usual factors like demographics or possible country and site effects. Analyses did not reveal definite hints on possible treatment relations. Subgroup analyses on more disease related confounders were performed as well. As a strong placebo effect could be observed, initial baseline symptoms might not have been severe enough to demonstrate a clear differentiation between treatments at the end of the trial. It was expected, that among all investigators, the specialists in otorhinolaryngology are most competent in making CRS diagnose.
Therefore, the subgroup was restricted to patients who were diagnosed and treated by specialists in otorhinolaryngology. Resulting factors for predicting a treatment differentiation were chosen as 1) specialist in otorhinolaryngology, 2) severe

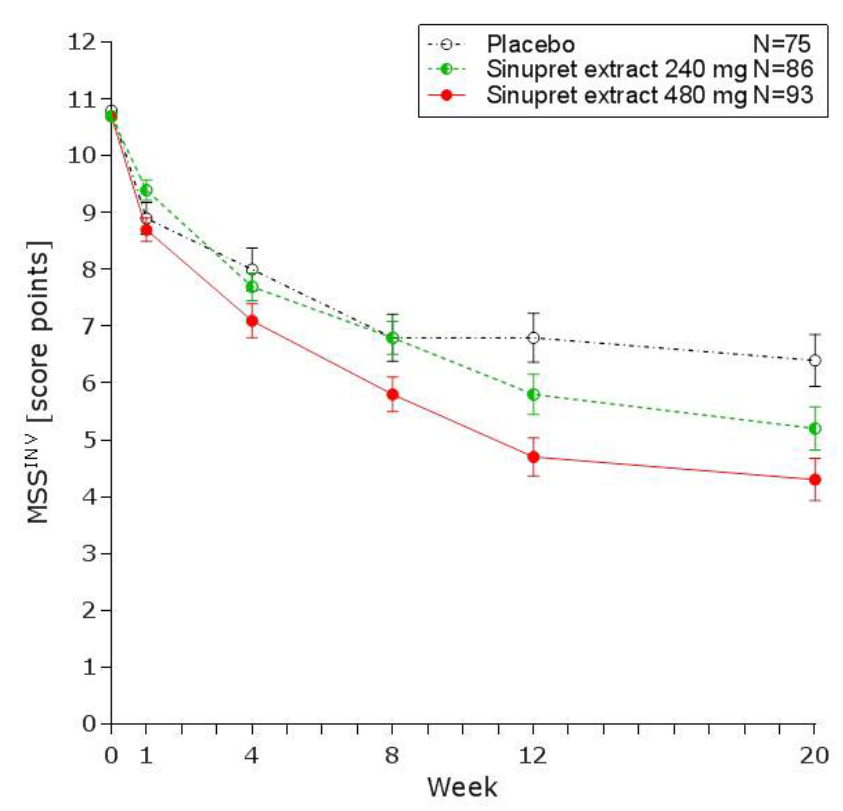

Figure 2. Mean MSSINV $( \pm$ SEM) in subgroup of patients who had a baseline MSS >9, had CRS persistence $>1$ year and were treated by a specialist in otorhinolaryngology (FAS). 
Table 2. Results of the WPAI:GH ${ }^{\mathrm{PAT}}$ in subgroup of patients who had a baseline MSS $>9$, had CRS persistence $>1$ year and were treated by a specialist in otorhinolaryngology (FAS).

\begin{tabular}{|c|c|c|c|c|}
\hline & $\begin{array}{l}\text { Sinupret extract } \\
240 \mathrm{mg}\end{array}$ & $\begin{array}{l}\text { Sinupret extract } \\
\qquad 480 \mathrm{mg}\end{array}$ & Placebo & $\begin{array}{l}\text { p-value vs. Placebo } \\
240 \mathrm{mg} / 480 \mathrm{mg}^{\mathrm{a}}\end{array}$ \\
\hline \multicolumn{5}{|c|}{ Percent overall work impairment due to health; Mean (SD) } \\
\hline & $\mathrm{N}=53$ & $\mathrm{~N}=52$ & $\mathrm{~N}=54$ & \\
\hline Baseline (day 1) & $52.3(22.5)$ & $51.3(20.3)$ & $52.0(22.4)$ & \\
\hline Visit 5 (week 8) & $40.0(19.4)$ & $29.5(15.4)$ & $40.0(25.7)$ & $0.6697 / 0.0036$ \\
\hline Visit 6 (week 12) & $34.8(19.1)$ & $21.9(18.2)$ & $41.9(28.2)$ & $0.0622 /<0.001$ \\
\hline Visit 7 (week 20) & $31.7(21.8)$ & $17.6(17.0)$ & $39.7(29.2)$ & $0.0282 /<0.001$ \\
\hline \multicolumn{5}{|c|}{ Percent work time missed due to health; Mean (SD) } \\
\hline & $\mathrm{N}=54$ & $\mathrm{~N}=52$ & $\mathrm{~N}=54$ & \\
\hline Baseline (day 1) & $5.4(17.4)$ & $2.0(5.7)$ & $5.6(16.7)$ & \\
\hline Visit 5 (week 8) & $4.6(16.6)$ & $3.2(15.4)$ & $4.0(19.0)$ & $0.6315 / 0.2938$ \\
\hline Visit 6 (week 12) & $3.1(15.3)$ & $8.0(24.4)$ & $5.7(19.9)$ & $0.2755 / 0.8777$ \\
\hline Visit 7 (week 20) & $3.7(17.4)$ & $3.5(15.7)$ & $0.5(2.4)$ & $0.2070 / 0.4676$ \\
\hline \multicolumn{5}{|c|}{ Percent impairment while working due to health; Mean (SD) } \\
\hline & $\mathrm{N}=55$ & $\mathrm{~N}=55$ & $\mathrm{~N}=58$ & \\
\hline Baseline (day 1) & $54.5(22.8)$ & $53.3(20.5)$ & $54.8(22.5)$ & \\
\hline Visit 5 (week 8) & $41.1(19.8)$ & $31.4(16.0)$ & $40.7(25.6)$ & $0.9580 / 0.0182$ \\
\hline Visit 6 (week 12) & $35.0(19.2)$ & $23.5(20.9)$ & $43.2(28.8)$ & $0.803 / 0.0003$ \\
\hline Visit 7 (week 20) & $31.1(21.0)$ & $17.8(18.7)$ & $38.9(29.4)$ & $0.0572 /<0.001$ \\
\hline \multicolumn{5}{|c|}{ Percent activity impairment due to health; Mean (SD) } \\
\hline & $\mathrm{N}=86$ & $\mathrm{~N}=93$ & $\mathrm{~N}=74$ & \\
\hline Baseline (day 1) & $56.0(20.6)$ & $54.8(20.8)$ & $60.8(20.1)$ & \\
\hline Visit 5 (week 8) & $41.1(21.5)$ & $36.4(21.2)$ & $44.5(24.9)$ & $0.7325 / 0.1328$ \\
\hline Visit 6 (week 12) & $32.9(21.0)$ & $28.5(22.5)$ & $44.5(28.5)$ & $0.0192 / 0.0019$ \\
\hline Visit 7 (week 20) & $32.5(23.2)$ & $25.4(24.4)$ & $38.1(26.8)$ & $0.4086 / 0.0135$ \\
\hline
\end{tabular}

a ANCOVA with 'MSS at Baseline (visit 2, day 1)' as a fixed effect covariate and 'Treatment' as a fixed effect. The centre effect described by the variable 'Medical Specialist' was included in the model as fixed effect.

symptoms at baseline, i.e. MSS > 9, and 3) a long persistence of the chronic disease, i.e. $>1$ year. Implementing these specifications, treatment effects with respect to the primary endpoint, i.e. average MSS INV at visit 5 (week 8) and visit 6 (week 12) was statistically significant in favor of Sinupret extract $480 \mathrm{mg}$ (Table 1) and showed a clinically relevant difference.

In detail, the between group difference (LSMEAN) was statistically significant for the high dose group compared to placebo after 12 weeks ( $\Delta 1.90$ score points, $\mathrm{p}=0.003)$ and 20 weeks $(\Delta$ 2.01 score points, $p=0.005$, Figure 2 ).

Moreover, analysis of the single items of the MSSINv over visit 5 (week 8) and visit 6 (week 12) revealed significantly superior treatment effects for the higher dose with respect to rhinorrhea anterior, headache and facial pain/pressure compared to placebo (Table 1). The significant effect persisted until visit 7 (week 20) for rhinorrhea anterior and facial pain/pressure (data not shown). Additionally, treatment effect of the $480 \mathrm{mg}$ was significantly different from placebo with respect to rhinorrhea posterior at visit 7 (week 20) (data not shown).

The patient assessment of the total symptom severity was significantly less at week $12(p=0.0012)$ and week $20(p=0.0046)$ when they received Sinupret extract $480 \mathrm{mg}$ compared to placebo.

Patients treated with the higher dose were significantly less 
Table 3. Number of patients with adverse events (SES).

\begin{tabular}{|c|c|c|c|c|}
\hline & $\begin{array}{l}\text { Sinupret extract } \\
240 \mathrm{mg}\end{array}$ & $\begin{array}{l}\text { Sinupret extract } \\
480 \mathrm{mg}\end{array}$ & Placebo & Total \\
\hline \multicolumn{5}{|c|}{ Patients with at least one adverse events during the treatment period (baseline to week 12) } \\
\hline & $N=318$ & $\mathrm{~N}=305$ & $N=306$ & $\mathrm{~N}=929$ \\
\hline Adverse Event & $172(54.1 \%)$ & $172(56.4 \%)$ & $184(60.1 \%)$ & $528(56.8 \%)$ \\
\hline Serious Adverse Event & $6(1.9 \%)$ & $3(1.0 \%)$ & $2(0.7 \%)$ & $11(1.2 \%)$ \\
\hline $\begin{array}{l}\text { Drug-related Adverse } \\
\text { Event }\end{array}$ & $14(4.4 \%)$ & $18(5.9 \%)$ & $12(3.9 \%)$ & $44(4.7 \%)$ \\
\hline $\begin{array}{l}\text { Discontinuation due to } \\
\text { Adverse Event }\end{array}$ & $5(1.6 \%)$ & $9(3.0 \%)$ & $6(2.0 \%)$ & $20(2.2 \%)$ \\
\hline \multicolumn{5}{|c|}{ Patients with at least one adverse events during the follow-up (week 12 to 20) } \\
\hline & $N=318$ & $\mathrm{~N}=305$ & $N=306$ & $\mathrm{~N}=929$ \\
\hline Adverse Event & $35(11.0 \%)$ & $25(8.2 \%)$ & $49(16.0 \%)$ & $109(11.7 \%)$ \\
\hline SAE & $2(0.6 \%)$ & $1(0.3 \%)$ & $2(0.7 \%)$ & $5(0.5 \%)$ \\
\hline \multicolumn{5}{|c|}{ Patients with most frequent drug-related adverse events during the treatment period (baseline to week 12) } \\
\hline & $\mathrm{N}=318$ & $\mathrm{~N}=305$ & $N=306$ & $\mathrm{~N}=929$ \\
\hline Upper abdominal pain & $4(1.3 \%)$ & $4(1.3 \%)$ & $2(0.7 \%)$ & $10(1.1 \%)$ \\
\hline Diarrhoea & $2(0.6 \%)$ & $1(0.3 \%)$ & $4(1.3 \%)$ & $7(0.8 \%)$ \\
\hline Headache & $1(0.3 \%)$ & $4(1.3 \%)$ & $2(0.7 \%)$ & $7(0.8 \%)$ \\
\hline Nausea & $3(0.9 \%)$ & $2(0.7 \%)$ & $1(0.3 \%)$ & $6(0.6 \%)$ \\
\hline Rash & $1(0.3 \%)$ & $2(0.7 \%)$ & $2(0.7 \%)$ & $5(0.5 \%)$ \\
\hline Abdominal pain & $1(0.3 \%)$ & $2(0.7 \%)$ & $0(0 \%)$ & $3(0.3 \%)$ \\
\hline Nasopharyngitis & $1(0.3 \%)$ & $2(0.7 \%)$ & $0(0 \%)$ & $3(0.3 \%)$ \\
\hline Pruritus & $1(0.3 \%)$ & $1(0.3 \%)$ & $1(0.3 \%)$ & $3(0.3 \%)$ \\
\hline \multicolumn{5}{|c|}{ Number of adverse events during the treatment period by intensity (baseline to week 12) } \\
\hline & 384 (100\%) & $376(100 \%)$ & 455 (100\%) & 1215 (100\%) \\
\hline Unknown & $2(0.5 \%)$ & $4(1.1 \%)$ & $0(0 \%)$ & $6(0.5 \%)$ \\
\hline Mild & $264(68.8 \%)$ & $267(71.0 \%)$ & 312 (68.6\%) & 843 (69.4\%) \\
\hline Moderate & $102(26.6 \%)$ & $97(25.8 \%)$ & $125(27.5 \%)$ & $324(26.7 \%)$ \\
\hline Severe & $16(4.2 \%)$ & $8(2.1 \%)$ & $18(4.0 \%)$ & 42 (3.5\%) \\
\hline
\end{tabular}

impaired compared to placebo-treated patients as could be taken from the WPAl:GH-items 'impairment while working due to health' at visits 5, 6 and 7 (i.e. weeks 8,12 and 20), as well as from the items 'overall work impairment due to health' and 'activity impairment due to health' at visit 6 and visit 7 (i.e. weeks 12 and 20). No significance was seen regarding 'percent work time missed due to health' (Table 2).

\section{Safety and tolerability}

In total, 1,215 adverse events were reported by 528 (57.0\%) patients during the treatment period and 150 adverse events by $109(11.7 \%)$ during the follow-up. Most of the events were of mild to moderate intensity. A relationship with the study drug was suspected in 14 patients (4.4\%) treated with Sinupret extract $240 \mathrm{mg}$; in 18 patients (5.9\%) treated with the $480 \mathrm{mg}$ and in 12 patients (3.9\%) who received placebo (Table 3).

The most common drug-related adverse events reported for the $240 \mathrm{mg}$, the $480 \mathrm{mg}$ and placebo were upper abdominal pain $(1.3 \%, 1.3 \%, 0.7 \%)$, diarrhoea $(0.6 \%, 0.3 \%, 1.3 \%)$ and headache $(0.3 \%, 1.3 \%, 0.7 \%)$.

Serious adverse events were noted in 11 patients (1.2\%) during the treatment period and in $5(0.5 \%)$ patients during the follow- 
up period. No causal relationship with the study medication was seen for all of these. One patient with 2 serious adverse events (liver and pancreatic disorder) reported during the follow-up period and a medical history of pancreatopathy since 2012 died during the post-treatment period ( $240 \mathrm{mg}$ group). The investigator considered all adverse events of this patient not related to the investigational drug.

Adverse events led to discontinuation in 20 patients (2.2\%). The incidence of adverse events leading to premature discontinuation was highest in the $480 \mathrm{mg}$ group (3.0\%) compared to the $240 \mathrm{mg}$ group and placebo ( $1.6 \%$ and $2.0 \%$, respectively). No medically relevant differences in laboratory parameters or vital signs were observed for patients treated with Sinupret extract compared to placebo.

Tolerability was assessed as 'very good' or 'good' by $89.6 \%, 88.5 \%$ and $91.2 \%$ of the investigators and by $86.8 \%, 86.2 \%$ and $82.9 \%$ of the patients ( $240 \mathrm{mg}, 480 \mathrm{mg}$ and placebo, respectively).

\section{Discussion}

Chronic rhinosinusitis is a disabling disease, for which single medications are so far not registered; therefore, surgery is considered frequently. The current clinical trial failed to show superiority over placebo with respect to the primary endpoint in the overall patient population. However, results of the secondary endpoints indicated that Sinupret extract improves symptoms in CRS patients, primarily facial pain or pressure, headache, and rhinorrhoea. Furthermore, daily treatment with Sinupret extract for about 12 weeks demonstrated a safety profile comparable to placebo.

The outcome of the primary endpoint was assumed to be a result of a strong placebo effect. This could be based on patients not really suffering from chronic rhinosinusitis (as patients were partially diagnosed by non-specialists), or suffering from symptoms not severe enough to demonstrate a clear difference between treatments. Moreover, a selection bias in cases of those individuals included in this study by non-otorhinologists could not be ruled out. Additional post-hoc sensitivity analyses identified possible modifiers of treatment success. Within a population with more severe and more persistent chronic disease, positive findings on the efficacy of Sinupret extract were substantiated. Patients with a baseline MSS above 9 , a persistence of the disease of more than 1 year, and those who were diagnosed and treated by a specialist in otorhinolaryngology, had a significant benefit from the treatment. The $480 \mathrm{mg}$ dose was considerably effective in most outcome measures. The averaged MSS ${ }^{\text {INV }}$ was significantly reduced at visit 5 (week 8) and 6 (week 12) compared to placebo. At visit 6 (week 12) and visit 7 (week 20) the difference was clinically relevant and statistically significant. Superior treatment effects were observed for rhinorrhoea anterior, headache and facial pain/pressure for the $480 \mathrm{mg}$ dose. Symptoms were less severe at visit 6 (week 12) and visit 7 (week
20) and the patients were less impaired with respect to work and daily activities. Consequently, the results of these subgroup analyses reveal that diagnosis by a specialist in otorhinolaryngology remarkably impacts the treatment effect. The latter result is also in line with an observational study on 91 patients with CRS who underwent consultation by a specialist in otorhinolaryngology ${ }^{(14)}$. The authors hypothesized that proper treatment by a specialist in otorhinolaryngology is a major factor for a good improvement of the disease in CRS patients. Therefore, specialist consultation possibly facilitates proper diagnosis and treatment initiation.

Persistence and severity of the disease also influenced the efficacy outcomes in the present study. The severity of the disease is already reflected in the EPOS 2012 guideline ${ }^{(1)}$. It differentiates between mild and moderate/severe disease in adults with CRS and its respective management scheme. Generally, treatment options recommended in the guideline focus on nasal saline irrigation and topical corticosteroids. Additionally, long-term antibiotics should be considered in more severe forms of a CRS (1). Nasal saline irrigation is intended to transport mucus and to soothe mucous membrane irritation, topical corticosteroids work against inflammation and subsequent swelling, and finally antibiotics target microbial biofilms in the sinuses. However, it is obvious that in particular the latter treatments often exhibit remarkable side effects. These may not be neglected even if their severity might only be less pronounced, as in the chronic state of the disease medication generally has to be administered for a longer time period or even as a long-term treatment.

As the herbal medicinal product Sinupret extract holds mucolytic/mucokinetic, anti-inflammatory, as well as anti-viral and antibacterial properties in ex vivo studies ${ }^{(15)}$, it ideally fits treatment recommendations for moderate/severe CRS. Major symptoms of CRS are identical with those recognised in the acute state of the disease. From an etiological point of view CRS is believed to develop from the acute state, and for this reason, similar symptoms like inflammation, oedema and breathing difficulties are present in both diseases. Therefore, one can suggest that a beneficial treatment for the acute state might be a good treatment option for the chronic state as well. Sinupret extract is supposed to be such a suitable herbal alternative for the treatment of acute and of chronic symptoms.

Efficacy and safety of Sinupret extract ( $480 \mathrm{mg} /$ day) for the treatment of acute rhinosinusitis could already be demonstrated in the randomized, controlled, double blind, multicenter ARhiSi-2 trial in $\mathbf{3 8 6}$ patients suffering from symptoms characterised by an acute onset ${ }^{(8)}$. Patients with symptom duration of $\leq 3$ days, mild to moderate facial pain and an MSS of $\geq 8$ and $\leq 12$ were treated for 15 days. Primary and secondary endpoints in the study were comparable to the current trial. The MSS improved progressively over the course of the 15-day-treatment in the active treatment and placebo by a mean of 7.4 to 2.4 with Sinu- 
pret extract and by a mean of 6.3 to 3.4 with placebo $(p=0.0008)$. Therefore, the trial resulted in clinically relevant, significant differences in favor of Sinupret extract and moreover provided symptom relief two days earlier than placebo. The tolerability of Sinupret extract in acute rhinosinusitis was judged as good. About $95 \%$ of the investigators and patients rated the tolerability to be 'very good' or 'good'. In the active treatment group only 13 out of 194 patients (6.7\%) prematurely terminated the study. Only 1 of that group left the study due to an adverse event $(0.5 \%)$ and 6 due to lack of efficacy (3.1\%).

The current CRS-02 study revealed comparable tolerability values. Sinupret extract treatment was assessed as 'very good' or 'good' by about $88 \%$ of the investigators and patients and in the two active treatment groups only 46 out of 622 patients (7.4\%) discontinued, 14 of them due to adverse events (2.3\%) and only $2(0.3 \%)$ due to a lack of efficacy. Hence, comparing both studies, it may be stated that the good safety and tolerability profile of Sinupret extract does not alter even with a longer treatment duration of about three months and that a deterioration is hardly to be expected. Not only the efficacy results of the post-hoc sensitivity analysis of the current trial, but the good safety and tolerability underline that Sinupret extract is a suitable treatment option also for CRS, especially for patients with a higher disease burden.

\section{Conclusion}

In summary, the present study has demonstrated that Sinupret extract can be recommended for the treatment of patients with chronic rhinosinusitis. Future trials are needed to substantiate the treatment effect. Selection of subgroups should be done with care e.g. maybe also considering new findings on inflammation endotypes via biomarker analysis ${ }^{(17,18)}$. This work shows, that the disease should persist long enough with a certain degree of severeness and patients should be diagnosed and treated by a specialist in otorhinolaryngology.

\section{Acknowledgement}

The authors would like to thank Bionorica SE, Neumarkt, Germany, for financial support and the investigators at all study centres for recruiting and monitoring the patients in compliance with the study protocol and Good Clinical Practice. The authors are also grateful to the patients for their participation and compliance with the study protocol. We would like to thank Annika Streithoff for biometrical support with the post-hoc sensitivity analyses and for critical review of the manuscript. We would also like to thank Stephanie Palm for coordinating the drafting and publication process and for her critical review. Finally, we would like to thank Dr. Karin Eichele (www.mediwiz.de) for her editorial assistance.

\section{Authorship contribution}

JP, Specialist in Otorhinolaryngology, was the Principal Investigator according to $\$ 40$ German Drug Law ('Leiter der klinischen Prüfung') of this multicentre study, and provided scientific advice for writing the trial protocol and publication manuscript. IS made substantial contributions to the conduct of the presented study and acquisition of data and provided scientific advice for the publication manuscript.

HS made substantial contributions to conception and design of the study, acquisition of data, and analysis and interpretation of data.

DAS and AA were substantially involved in the interpretation of data, provided scientific advice for writing the publication manuscript, and gave final approval of the version to be published. SM and MW were involved in drafting the manuscript, revising it critically for important intellectual content, ensuring that questions related to the accuracy or integrity of the work are resolved. CB was the coordinating investigator of the CRS-02 trial, made substantial contributions to conception, design, and to the conduct of the study. He gave scientific advice, contributed to the interpretation of data and was involved in drafting and critically reviewing the manuscript.

\section{Conflict of interest}

D. Abramov-Sommariva, A. Ammendola, S. Mitzenheim, $\mathrm{H}$. Steindl and M. Wonnemann are employees of Bionorica SE, Germany. C. Bachert was speaker and consultant for Bionorica SE. All other authors have no conflict of interest.

\section{References}

1. Fokkens WJ, Lund VJ, Mullol J, Bachert C, Alobid I, Baroody F, et al. EPOS 2012 European position paper on rhinosinusitis and nasal polyps 2012. A summary for otorhinolaryngologists. Rhinology. 2012;50(1):1-12.

2. Prokopakis EP, Hellings PW, Velegrakis GA, Kawauchi H. From ancient Greek medicine to EP3OS. Rhinology 2010; 48(3):265-72.

3. Prokopakis EP, Vlastos $I M$, Ferguson BJ, Scadding G, Kawauchi H, Georgalas C, Papadopoulos N, Hellings PW. SCUAD and chronic rhinosinusitis. Reinforcing hypothesis driven research in difficult cases. Rhinology. 2014; 52(1):3-8

4. Fokkens WJ, Lund VJ, Mullol J, Bachert C, Alobid I, Baroody F, et al. European Position Paper on Rhinosinusitis and Nasal Polyps 2012. Rhinol Suppl. 2012(23): 1-298.

5. Hastan D, Fokkens WJ, Bachert C, et al. Chronic rhinosinusitis in Europe--an underestimated disease. A GA²LEN study. Allergy. 2011 66(9):1216-23.

6. Smith KA, Orlandi RR, Rudmik L. Cost of adult chronic rhinosinusitis: A systematic review. Laryngoscope. 2015;125(7):1547-56.

7. Rudmik L, Smith TL, Schlosser RJ, Hwang $\mathrm{PH}$, Mace JC, Soler ZM. Productivity costs in patients with refractory chronic rhinosinusitis. Laryngoscope. 2014;124(9):2007-12.

8. Jund $\mathrm{R}$, Mondigler M, Steind $\mathrm{H}$, Stammer H, Stierna P, Bachert C, et al. Clinical efficacy of a dry extract of five herbal drugs in acute viral rhinosinusitis. Rhinology. 2012:50(4):417-26.

9. World Medical Association (WMA): WMA Declaration of Helsinki - Ethical principles for medical research involving human sub- 
jects. Adopted by the 48th WMA Genera Assembly, Somerset West, October 1996.

10. Revicki D, Margolis M, Thompson C, Meltzer E, Sandor D, Shaw J. Major Symptom Score Utility Index for patients with acute rhinosinusitis. Am J Rhinol Allery. 2011; 25(3):99106.

11. ICH Topic E6 (R1), Step 5 Note for Guidance on Good Clinical Practice (CPMP/ $\mathrm{ICH} / 135 / 95)$. Final approval by CPMP: July 1996 / Date for coming into operation: January 1997; Post Step Errata (linguistic minor corrections): July 2002.

12. Benjamini $Y$ and Hochberg Y. Controlling the False Discovery Rate: A Practical and Powerful Approach to Multiple Testing. J.R.Statist.Soc.B. 1995;57(1):289-300.

13. SAS Institute Inc. SAS/STAT 9.2 SAS User's Guide. 9.2 ed. Cary. North Carolina USA2002-2008.

14. Lange $B$, Thilsing $T$, Baelum J, Pedersen
OF, Holst R, Kjeldsen AD. Do patients with chronic rhinosinusitis benefit from consultation with an ENT-doctor? Acta Otolaryngologica. 2015;135(7):706-12.

15. Passali D, Cambi J, Passali FM, Bellussi LM. Phytoneering: a new way of therapy for rhinosinusitis. Acta Otorhinolaryngol Ital. 2015;35(1):1-8.

16. Richstein A, Mann W. [Treatment of chronic sinusitis with Sinupret]. Ther Ggw. 1980;119(9):1055-60.

17. Tomassen P, Vandeplas G, Van Zele T, et al. Inflammatory endotypes of chronic rhinosinusitis based on cluster analysis of biomarkers. J Allergy Clin Immunol. 2016, 137(5): 1449-1456.e4.

18. Fokkens WJ. Phenotyping, endotyping and clinical decision-making. Rhinology 2016 54(2):97-98.

\section{Dr. Meinolf Wonnemann}

Senior Manager Translational

Research, M.Sc.

Clinical Research International

Bionorica SE

Kerschensteinerstraße 11-15

92318 Neumarkt

Deutschland

Tel: +49 (0) $9181231-7086$

Fax: +49 (0) 9181 231-67086

\section{E-mail:}

meinolf.wonnemann@bionorica.de 